\title{
EVALUACIÓN DE UN PROCESO ANÓXICO-AEROBIO-REACTOR BIOLÓGICO DE MEMBRANA CON ALTO CONTENIDO DE NITRÓGENO
}

\author{
Evaluation of an anoxic-aerobic MBR process with high nitrogen content
}

\author{
Miguel Ángel ESPINOSA-RODRÍGUEZ*, Raúl DELGADO-DELGADO \\ y Antonio HIDALGO-MILLÁN
}

Programa Académico de Ingeniería Química, Universidad Autónoma de Nayarit, Ciudad de la Cultura Amado Nervo s/n, 63155 Tepic, Nayarit, México

*Autor para correspondencia: emiguel@uan.edu.mx

(Recibido: diciembre 2018; aceptado: agosto 2019)

Palabras clave: desnitrificación, GPS-X, nitrificación

\section{RESUMEN}

La industria de elaboración de harinas de pescado genera aguas residuales con alto contenido de carga orgánica y nitrógeno que se deben tratar antes de descargarse a un cuerpo receptor. El objetivo de este trabajo de investigación fue evaluar el sistema de tratamiento biológico (anóxico-aerobio-reactor biológico de membrana) que opera una empresa dedicada a la elaboración de harinas de sardina y atún. Este sistema de tratamiento presenta reacciones de nitrificación y desnitrificación incompletas, lo cual ocasiona que la remoción de nitrógeno sea deficiente e incumpla con los límites máximos permitidos de descarga. El trabajo se desarrolló con escenarios de evaluación, ampliación y modificación del proceso a través de la simulación con el programa GPS$\mathrm{X}$. Los resultados mostraron desnitrificación deficiente en el reactor anóxico, por lo que se requiere un incremento de nitrificación en el reactor aerobio. El reactor biológico de membrana (MBR, por sus siglas en inglés) cumplió sólo con el objetivo de filtración. Con base en ello se simularon escenarios de cambios en el proceso incluyendo la eliminación del MBR y la adecuación de un clarificador secundario, el cual ayudaría en el proceso de desnitrificación por sus condiciones anóxicas. Con el sistema actual se tiene una remoción de nitrógeno total del $49.5 \%$, mientras que con la adecuación del sistema se alcanzó una eficiencia del $94.7 \%$.

Key words: denitrification, GPS-X, nitrification

\begin{abstract}
The fishmeal processing industry generates wastewater with high content of organic load and nitrogen, therefore it should be treated before its discharge into a receiver body. The objective of this research work was to evaluate a biological treatment system (anoxic-aerobic-membrane biological reactor) operated by a company dedicated to the processing of sardine and tuna meal. This treatment system presents incomplete nitrification and denitrification reactions, which cause deficient removal of nitrogen and non-compliance with the maximum allowable discharge limits. The work was developed with scenarios for evaluation, extension and modification of this process through a simulation with the GPS-X software. The results showed deficient denitrification in
\end{abstract}


the anoxic reactor, therefore increased nitrification is required in the aerobic reactor, and the membrane biological reactor (MBR) only fulfilled the objective of filtration. On this basis, scenarios were simulated for modifications in the process, including the elimination of the MBR and the adequacy of a secondary clarifier, which would help in the denitrification process due to its anoxic conditions. The actual system it has a total effectiveness $49.5 \%$ for nitrogen removal, while the adequacy of the system improved the efficiency to $94.7 \%$.

\section{INTRODUCCIÓN}

Las actividades de procesamiento de pescado generan grandes cantidades de residuos orgánicos y subproductos procedentes de las partes no comestibles del pescado. Al ser una fuente rica de aminoácidos, estos residuos pueden transformarse en subproductos (harina de pescado). El procesamiento de pescado requiere bastante agua para lavado, limpieza y el almacenamiento, antes y durante el procesamiento. Las aguas residuales generadas en el proceso tienen un alto contenido orgánico (sangre, tejidos y proteínas disueltas), además de un elevado contenido de nitrógeno (sangre) y fósforo (Pratyush et al. 2014).

El pescado fresco es susceptible al deterioro, ya que ciertas reacciones químicas, actividad enzimática endógena y crecimiento microbiano alteran su composición, originando compuestos químicos volátiles. Durante la alteración microbiana se forman aminas volátiles y no volátiles por descarboxilación y desaminación de los aminoácidos y las bases orgánicas. La determinación de bases volátiles, entre las que se encuentran amoniaco, mono, di y trimetilamina, histamina, etc., se hace en función del contenido de nitrógeno básico volátil total (NBVT), índice que se considera representativo del grado de frescura del pescado. El NBVT incluye las bases nitrogenadas volátiles, como trimetilamina (producida por el deterioro bacteriano), dimetilamina (producida por enzimas autolíticas durante la congelación), amoniaco (producido por desaminación de aminoácidos y catabolitos de nucleotidos) y otros compuestos nitrogenados básicos volátiles asociados con el deterioro de los productos pesqueros (Bhadra et al. 2015).

En este trabajo, se evaluó el sistema biológico de tratamiento de aguas residuales (anóxico-aerobioreactor biológico de membrana) de una empresa dedicada a la elaboración de harinas de sardina y atún, con ayuda del simulador GPS-X. El agua residual generada por esta empresa posee alto contenido de $\mathrm{DBO}_{5}(5831 \mathrm{mg} / \mathrm{L})$ y nitrógeno total $(1697 \mathrm{mg} / \mathrm{L})$. La eficiencia del tratamiento en el sistema biológico indicó baja remoción de nitrógeno, incumpliendo con los límites máximos permisibles de descarga (SEMARNAT 1996).

El programa GPS-X es una herramienta que realiza modelación matemática, simulación, optimización y evaluación de plantas de tratamiento de aguas residuales en estado estacionario y dinámico. Optimiza también sistemas de control avanzado y predice la calidad del efluente en condiciones variables (Hydromantis 2014). Muchos beneficios significativos se asocian con el uso de simuladores en el análisis, diseño y operación de sistemas de tratamiento de aguas residuales (van Loosdrecht et al. 2015, Espinosa et al. 2019).

En este contexto, el objetivo de esta investigación fue realizar una evaluación del sistema biológico de tratamiento de aguas residuales de la empresa en estudio, con ayuda del programa GPS-X, en cuanto a remoción de carga orgánica, nitrificación y desnitrificación, y con ello determinar el problema de la baja remoción del nitrógeno.

\section{MATERIAL Y MÉTODOS}

\section{Planta de tratamiento de aguas residuales}

La planta de tratamiento de aguas residuales (PTAR) tiene una capacidad nominal de $300 \mathrm{~m}^{3} / \mathrm{d}$, aunque en la actualidad se tratan solamente $190 \mathrm{~m}^{3} / \mathrm{d}$. El tratamiento comprende tres etapas: pretratamiento, tratamiento biológico y tratamiento terciario. La primera etapa está conformada, en ese orden, por un canal de recepción, tamiz tipo tornillo, cárcamo de bombeo, rotafiltro (hidrotamiz), tanque de homogeneización, floculador y flotación con aire disuelto (FAD). La etapa de tratamiento biológico motivo de este estudio comprende un reactor anóxico de $133 \mathrm{~m}^{3}$, un primer reactor aerobio tipo mezcla completa (CSTR1) de $300 \mathrm{~m}^{3}$, un segundo reactor aerobio tipo mezcla completa (CSTR2) de $330 \mathrm{~m}^{3}$ y finalmente un reactor biológico de membrana (MBR, por sus siglas en inglés) de tipo sumergido de $127 \mathrm{~m}^{3}$. La aireación en los reactores aerobios se realiza a través de difusores de burbuja fina. El MBR tiene un área 
superficial de membrana sumergida de $1920 \mathrm{~m}^{2}$, y su filtrado-retrolavado se realiza automáticamente cumpliendo un ciclo de $510 \mathrm{~s}$ ( $8.5 \mathrm{~min})$. Finalmente, la etapa de tratamiento terciario, en la cual se procesa el efluente permeado del MBR, consiste, en ese orden, en desinfección con ozono, adsorción con carbón activado y ósmosis inversa. El efluente final es utilizado para riego de áreas verdes y se descarga también hacia el mar. Los lodos generados se tratan en un decantador de lodos y luego son enviados a PTAR municipales por medio de pipas.

\section{Caracterización del agua residual en influente, efluente y lodos}

La caracterización del agua residual en el influente al reactor anóxico, la caracterización de sólidos en el sistema biológico (anóxico-aerobio-MBR) y la caracterización del efluente permeado del MBR, se obtuvieron a partir del historial de análisis (siete meses) de la PTAR. Por otra parte, se realizó una campaña de muestreo compuesto diurno de cinco días (muestreo cada $2 \mathrm{~h}$ ), para obtener los datos necesarios para el fraccionamiento de la DQO y nitrógeno con el programa Influent Advisor, el cual basa su caracterización en filtraciones a $0.45 \mu \mathrm{m}$. Los parámetros de demanda bioquímica de oxígeno $\left(\mathrm{DBO}_{5}\right)$, demanda química de oxígeno total y soluble (DQO), sólidos suspendidos totales (SST), sólidos suspendidos volátiles (SSV), nitrógeno total Kjeldahl (NTK), nitrógeno total y soluble $\left(\mathrm{N}_{\mathrm{TOT}}\right.$ ), nitrógeno amoniacal total y soluble $\left(\mathrm{N}-\mathrm{NH}_{3}\right)$, nitratos $\left(\mathrm{NO}_{3}{ }^{-}\right)$, nitritos $\left(\mathrm{NO}_{2}{ }^{-}\right)$y alcalinidad (como $\left.\mathrm{CaCO}_{3}\right)$, se analizaron siguiendo los procedimientos de métodos estándar para el análisis de aguas residuales (APHA 2005). Los parámetros referidos como solubles se analizaron filtrando la muestra con un filtro de $0.45 \mu \mathrm{m}$. El oxígeno disuelto (OD), la temperatura y el $\mathrm{pH}$ se analizaron en campo con un equipo portátil Thermo Scientific Marca Orión 3 Star. La DQO, NTK, N-NH $3, \mathrm{~N}_{\text {TOT }}, \mathrm{NO}_{3}{ }^{-}$y $\mathrm{NO}_{2}{ }^{-}$fueron analizados con un digestor Hach DRB 200 y un espectrofotómetro Hach DR 2800.

\section{Fraccionamiento de la DQO y nitrógeno con In- fluent Advisor}

La caracterización del influente al reactor anóxico se realizó con base a lo solicitado por el programa Influent Advisor con el modelo Codstates preinstalado en el programa GPS-X, el cual convierte los datos obtenidos en campaña en un juego de fracciones de sustrato orgánico (fraccionamiento de DQO) y nitrógeno (fraccionamiento del nitrógeno). El programa Influent Advisor está compuesto por tres columnas básicas: en la primera (datos de entrada) se ingresaron los resultados obtenidos a partir de la caracterización del influente al anóxico tales como DQO total, NTK total, $\mathrm{N}_{-} \mathrm{NH}_{3}$ soluble, $\mathrm{OD}$, alcalinidad y algunos coeficientes estequiométricos como icv (DQO particulada/SSV), ivt (SSV/SST), frsi (fracción de DQO inerte soluble), frxi (fracción de DQO inerte particulada), frsnh (N-NH 3 soluble/NTK soluble) .Las fracciones de nutrientes se tomaron "por defecto". El valor de frss (fracción de DQO rápidamente biodegradable) y fbod $\left(\mathrm{DBO}_{5} / \mathrm{DBO}_{\mathrm{u}}\right)$ se obtuvieron por iteración hasta que coincidiera la $\mathrm{DBO}_{5}$ medida en el influente con el valor calculado por Influent Advisor. En la segunda columna (variables de estado) se obtienen los resultados del fraccionamiento de la DQO $\left(\mathrm{S}_{\mathrm{I}}\right.$, $\left.\mathrm{S}_{\mathrm{S}}, \mathrm{X}_{\mathrm{I}}, \mathrm{X}_{\mathrm{S}}\right)$ y del $\mathrm{N}\left(\mathrm{S}_{\mathrm{NH}}, \mathrm{S}_{\mathrm{ND}}, \mathrm{X}_{\mathrm{ND}}\right)$. Por último, en la tercera columna se obtienen resultados de algunas variables compuestas.

\section{Escenarios de simulación}

Los escenarios de simulación se desarrollaron en cuatro etapas.

\section{Primera etapa}

Se desarrollaron escenarios para el sistema de tratamiento biológico en estudio, con el fin de observar su comportamiento en cuanto a remoción de carga orgánica y nitrógeno. Para ello se introdujeron los datos de caracterización del agua residual y lodos obtenidos en la campaña de muestreo, así como el dimensionamiento actual de cada uno de los reactores. A través de la simulación se observó la desnitrificación en el reactor anóxico, el proceso de nitrificación en los reactores aerobios de tipo mezcla completa (CSTR) y la calidad del efluente permeado en el MBR.

\section{Segunda etapa}

Se simularon escenarios para el sistema de tratamiento biológico actual, variando parámetros importantes en el proceso de nitrificación-desnitrificación, tales como $\mathrm{pH}$, temperatura, OD, alcalinidad, concentración de sólidos suspendidos en cada uno de los reactores, flujo de recirculación (QRAS) y purga (QwAs), con el fin de observar la eficiencia de remoción de carga orgánica y nitrógeno.

\section{Tercera etapa}

El sistema de tratamiento biológico actual fue modificado. Se construyó un nuevo reactor anóxico, se amplió el CSTR y sólo se dejó como tal el MBR. La ampliación del tratamiento aerobio consistió únicamente en cambiar el reactor anóxico por un CSTR, quedando entonces tres CSTR con las mismas 
dimensiones actuales para evitar costos mayores. En esta etapa se simularon varios escenarios, con los datos de caracterización del agua residual obtenidos en campaña en el influente al anóxico, y variando la concentración de sólidos en cada uno de los reactores (anóxico-CSTR-MBR), así como el QRAS y Qwas. Para este modelo, se consideró el mejor escenario de operación en cuanto al OD según los resultados obtenidos en la segunda etapa. La temperatura, el pH y la alcalinidad se mantuvieron con las condiciones de operación normal de la PTAR actual.

\section{Cuarta etapa}

El sistema de tratamiento biológico actual se modificó. Se construyó un nuevo reactor anóxico, se amplió el tren de tratamiento aerobio (CSTR) y se cambió el MBR por un clarificador secundario (CS). La ampliación del tratamiento aerobio consistió sólo en cambiar el reactor anóxico por un CSTR, quedando entonces tres CSTR con las mismas dimensiones actuales para evitar costos mayores. Para este último modelo se seleccionaron los mejores escenarios de las etapas anteriores hasta llegar a la optimización del sistema de tratamiento.

\section{RESULTADOS Y DISCUSIÓN}

\section{Caracterización del agua residual en influente, efluente y lodos}

En el cuadro I se presentan los resultados promedio obtenidos en siete meses de caracterización del influente al reactor anóxico. En el cuadro II se presenta la calidad del efluente tratado en el proceso biológico, que sirve como base para analizar la eficiencia de remoción de los contaminantes en siete meses de operación de la planta de tratamiento. El muestreo simple y el análisis se realizaron seis días a la semana. Como se observa en el cuadro I, la concentración de carga orgánica (DQO) es muy elevada, al igual que la concentración de nitrógeno total $\left(\mathrm{N}_{\mathrm{TOT}}\right)$. Estas cargas de contaminantes que ingresan al tratamiento biológico son eliminadas en cierta proporción, siendo la DQO la que más se remueve (95.4 \%), no así el $\mathrm{N}_{\text {TOт }}$ у el $\mathrm{P}_{\text {TOт, }}$, cuyas remociones son del 52.2 y $44 \%$, respectivamente. De acuerdo a estos resultados, se observa también que se lleva a cabo una nitrificación, ya que hay una reducción del $\mathrm{N}-\mathrm{NH}_{3}$ en un $49.6 \%$ y se consume alcalinidad en un $45 \%$ por oxidación del amonio. Por el contrario, en el

CUADRO I. CARACTERIZACIÓN DEL AGUA RESIDUAL (INFLUENTE) DE SIETE MESES DE MUESTREO

\begin{tabular}{lrrrrrrrr}
\hline Parámetros & Ene & Feb & Mar & Abr & May & Jun & Jul & Promedio \\
\hline Temperatura $\left({ }^{\circ} \mathrm{C}\right)$ & 39.0 & 39.0 & 38.0 & 35.0 & 36.0 & 39.0 & 38.0 & 38 \\
pH & 7.0 & 7.0 & 6.9 & 6.8 & 6.7 & 6.8 & 7.0 & 6.9 \\
Alcalinidad $(\mathrm{mg} / \mathrm{L})$ como $\mathrm{CaCO}_{3}$ & 4356 & 4812 & 4400 & 4011 & 3579 & 3873 & 3527 & 4080 \\
$\mathrm{SST}(\mathrm{mg} / \mathrm{L})$ & 74 & 105 & 100 & 98 & 132 & 185 & 175 & 124 \\
DQO total $(\mathrm{mg} / \mathrm{L})$ & 7212 & 8505 & 8037 & 7138 & 8327 & 7366 & 7517 & 7729 \\
P total $(\mathrm{mg} / \mathrm{L})$ & 238 & 193 & 220 & 159 & 141 & 98 & 177 & 175 \\
$\mathrm{~N}_{-} \mathrm{NH}_{3}(\mathrm{mg} / \mathrm{L})$ & 1166 & 1261 & 1207 & 1110 & 1251 & 1184 & 1108 & 1184 \\
$\mathrm{~N}_{\text {TOT }}(\mathrm{mg} / \mathrm{L})$ & 1392 & 1443 & 1310 & 1358 & 1359 & 1385 & 1281 & 1361 \\
\hline
\end{tabular}

pH: potencial de hidrógeno, $\mathrm{CaCO}_{3}$ : carbonato de calcio, SST: sólidos suspendidos totales, DQO: demanda química de oxígeno, $\mathrm{P}$ : fósforo, $\mathrm{N}-\mathrm{NH}_{3}$ : nitrógeno amoniacal, $\mathrm{N}_{\mathrm{TOT}}$ : nitrógeno total

CUADRO II. CARACTERIZACIÓN DEL AGUA RESIDUAL (EFLUENTE) DE SIETE MESES DE MUESTREO

\begin{tabular}{lcccccccc}
\hline Parámetros & Ene & \multicolumn{1}{c}{ Feb } & Mar & Abr & May & Jun & Jul & Promedio \\
\hline Temperatura $\left({ }^{\circ} \mathrm{C}\right)$ & 39.0 & 40.0 & 37.0 & 37.0 & 40.0 & 41.0 & 39.0 & 39.0 \\
pH & 7.9 & 8.5 & 7.1 & 8.2 & 8.2 & 8.4 & 8.6 & 8.1 \\
Alcalinidad (mg/L) como $\mathrm{CaCO}_{3}$ & 1805 & 1846 & 1636 & 1709 & 2839 & 2890 & 2983 & 2244 \\
SST (mg/L) & 5 & 6 & 5 & 9 & 16 & 13 & 13 & 10 \\
DQO total (mg/L) & 307 & 332 & 376 & 472 & 297 & 403 & 289 & 354 \\
P total (mg/L) & 122 & 103 & 114 & 142 & 73 & 40 & 92 & 98 \\
$\mathrm{~N}_{-} \mathrm{NH}_{3}(\mathrm{mg} / \mathrm{L})$ & 331 & 577 & 356 & 650 & 594 & 870 & 803 & 597 \\
$\mathrm{~N}_{\mathrm{TOT}}(\mathrm{mg} / \mathrm{L})$ & 345 & 618 & 420 & 707 & 614 & 940 & 908 & 650 \\
\hline
\end{tabular}

pH: potencial de hidrógeno, $\mathrm{CaCO}_{3}$ : carbonato de calcio, SST: sólidos suspendidos totales, DQO: demanda química de oxígeno, $\mathrm{P}$ : fósforo, $\mathrm{N}_{-} \mathrm{NH}_{3}$ : nitrógeno amoniacal, $\mathrm{N}_{\text {TOT: }}$ nitrógeno total 
proceso de desnitrificación disminuye la alcalinidad, por lo cual aumenta el $\mathrm{pH}$ en el efluente (Espinosa et al. 2014, 2019)

Respecto de la temperatura, el $\mathrm{pH}$ y la alcalinidad, los cuales promueven el proceso de nitrificación, en el cuadro III se presentan datos promedio estadísticos obtenidos en el laboratorio de la PTAR en estudio durante siete meses. A temperaturas mayores de $15^{\circ} \mathrm{C}$ y $\mathrm{pH}$ entre 7.5 y 8.5 , se alcanzan tasas de nitrificación constantes (Espinosa et al. 2014, 2019).

CUADRO III. CARACTERÍSTICAS FÍSICAS Y QUÍMICAS EN REACTORES DEL SISTEMA BIOLÓGI$\mathrm{CO}$

\begin{tabular}{lcccc}
\hline Parámetros & $\begin{array}{c}\text { Reactor } \\
\text { anóxico }\end{array}$ & CSTR1 & CSTR2 & MBR \\
\hline Temperatura $\left({ }^{\circ} \mathrm{C}\right)$ & 38 & 40 & 40 & 39 \\
pH & 8.0 & 8.0 & 8.0 & 8.0 \\
Oxígeno disuelto $(\mathrm{mg} / \mathrm{L})$ & 0.29 & 3.9 & 3.9 & 3.9 \\
Alcalinidad $(\mathrm{mg} / \mathrm{L})$ & 3073 & 2442 & 2442 & 2231 \\
SST $(\mathrm{mg} / \mathrm{L})$ & 10454 & 7783 & 7783 & 7783 \\
SSV $(\mathrm{mg} / \mathrm{L})$ & 8383 & 6305 & 6305 & 6305 \\
\hline
\end{tabular}

pH: potencial de hidrógeno, SST: sólidos suspendidos totales, SSV: sólidos suspendidos volátiles

Los datos del cuadro III representan parámetros importantes de operación con los cuales trabaja normalmente la planta. La concentración de SST en un MBR con membranas del tipo sumergido varía típicamente de 10000 a $25000 \mathrm{mg} / \mathrm{L}$ (Radjenović et al. 2008), o de 5000 a $20000 \mathrm{mg} / \mathrm{L}$ (Tchobanoglous et al. 2003). Según datos técnicos del MBR proporcionados por el fabricante, el contenido de SST recomendado es de $12000 \mathrm{mg} / \mathrm{L}$. Por otra parte, un CSTR opera en un rango de 1500 a $4000 \mathrm{mg} / \mathrm{L}$ de SST (Tchobanoglous et al. 2003).

En el cuadro IV se presentan los resultados de cinco días de muestreo compuesto diurno de la caracterización del agua residual (influente al anóxico) necesarios para el programa Influent Advisor. Como se observa, se realizaron análisis de concentraciones solubles de algunos parámetros, ya que así lo solicita el programa. Estos análisis no se realizan en el laboratorio de la PTAR en estudio. En el cuadro V se presentan los resultados de cinco días de muestreo compuesto diurno de la caracterización del agua residual (efluente permeado del MBR).

Los resultados del cuadro IV se ingresaron al programa Influent Advisor, y los del cuadro $\mathbf{V}$ se tomaron como referencia para comparar la calidad del efluente con la NOM-001-SEMARNAT-1996 (SEMARNAT 1996) en cuanto a $\mathrm{SST}_{\text {, }} \mathrm{DBO}_{5}$ y $\mathrm{N}_{\mathrm{TOT}}$ (Cuadro VI).

\section{Fraccionamiento de la DQO y nitrógeno con In- fluent Advisor}

Los resultados del programa Influent Advisor (Fig. 1) determinaron el fraccionamiento de la DQO

CUADRO IV. CARACTERIZACIÓN DEL INFLUENTE A REACTOR ANÓXICO

\begin{tabular}{|c|c|c|c|c|c|c|}
\hline Parámetros & $3 / 08 / 15$ & $4 / 08 / 15$ & $5 / 08 / 15$ & $6 / 08 / 15$ & $7 / 08 / 15$ & Promedio \\
\hline $\mathrm{OD}(\mathrm{mg} / \mathrm{L})$ & 0.48 & 0.52 & 0.54 & 0.50 & 0.54 & 0.52 \\
\hline DQO total $(\mathrm{mg} / \mathrm{L})$ & 9360 & 8990 & 9425 & 9600 & 8500 & 9175 \\
\hline DQO soluble $(\mathrm{mg} / \mathrm{L})$ & 5528 & 6522 & 5625 & 6250 & 6200 & 6025 \\
\hline $\mathrm{DBO}_{5}(\mathrm{mg} / \mathrm{L})$ & 5995 & 5950 & 5810 & 6177 & 5223 & 5831 \\
\hline $\mathrm{SST}(\mathrm{mg} / \mathrm{L})$ & 135 & 159 & 123 & 140 & 178 & 147 \\
\hline $\mathrm{SSV}(\mathrm{mg} / \mathrm{L})$ & 25 & 40 & 23 & 20 & 52 & 32 \\
\hline $\mathrm{N}-\mathrm{NH}_{3}(\mathrm{mg} / \mathrm{L})$ & 1200 & 1275 & 1000 & 1300 & 1410 & 1237 \\
\hline $\mathrm{N}-\mathrm{NH}_{3}$ soluble $(\mathrm{mg} / \mathrm{L})$ & 829 & 1131 & 750 & 890 & 1300 & 980 \\
\hline $\mathrm{N}$ total $(\mathrm{mg} / \mathrm{L})$ & 1650 & 1745 & 1690 & 1700 & 1700 & 1697 \\
\hline $\mathrm{N}$ total soluble $(\mathrm{mg} / \mathrm{L})$ & 1438 & 1582 & 1450 & 1500 & 1580 & 1510 \\
\hline $\mathrm{N}-\mathrm{NO}_{2}{ }^{-}(\mathrm{mg} / \mathrm{L})$ & 10.2 & 8.2 & 12.8 & 13.1 & 9.0 & 10.7 \\
\hline $\mathrm{N}-\mathrm{NO}_{2}{ }^{-}$soluble $(\mathrm{mg} / \mathrm{L})$ & 8.9 & 7.5 & 11.3 & 11.9 & 6.8 & 9.3 \\
\hline $\mathrm{N}-\mathrm{NO}_{3}{ }^{-}(\mathrm{mg} / \mathrm{L})$ & 18.0 & 15.6 & 20.3 & 20.4 & 16.5 & 18.2 \\
\hline $\mathrm{N}-\mathrm{NO}_{3}{ }^{-}$soluble $(\mathrm{mg} / \mathrm{L})$ & 16.8 & 13.9 & 17.8 & 16.9 & 13.2 & 15.7 \\
\hline NTK total $(\mathrm{mg} / \mathrm{L})$ & 1622 & 1723 & 1658 & 1669 & 1676 & 1670 \\
\hline NTK soluble $(\mathrm{mg} / \mathrm{L})$ & 1420 & 1560 & 1429 & 1465 & 1558 & 1486 \\
\hline
\end{tabular}

OD: oxígeno disuelto, DQO: demanda química de oxígeno, $\mathrm{DBO}_{5}$ : demanda bioquímica de oxígeno a los 5 días, SST: sólidos suspendidos totales, SSV: sólidos suspendidos volátiles, $\mathrm{N}_{-} \mathrm{NH}_{3}$ : nitrógeno amoniacal, $\mathrm{N}$ : nitrógeno, ${\mathrm{N}-\mathrm{NO}_{2}}^{-}$: nitrógeno como nitritos, ${\mathrm{N}-\mathrm{NO}_{3}}^{-}$: nitrógeno como nitratos, $\mathrm{NTK}$ : nitrógeno total Kjeldahl 
CUADRO V. CARACTERIZACIÓN DEL EFLUENTE PERMEADO DEL REACTOR BIOLÓGICO DE MEMBRANA (MBR)

\begin{tabular}{|c|c|c|c|c|c|c|}
\hline Parámetros & $3 / 08 / 15$ & $4 / 08 / 15$ & $5 / 08 / 15$ & $6 / 08 / 15$ & $7 / 08 / 15$ & Promedio \\
\hline DQO total $(\mathrm{mg} / \mathrm{L})$ & 290 & 275 & 280 & 300 & 270 & 283 \\
\hline DQO soluble $(\mathrm{mg} / \mathrm{L})$ & 260 & 195 & 245 & 190 & 250 & 228 \\
\hline $\mathrm{DBO}_{5}(\mathrm{mg} / \mathrm{L})$ & 5.6 & 5.4 & 4.8 & 7.7 & 4 & 5.5 \\
\hline $\mathrm{SST}(\mathrm{mg} / \mathrm{L})$ & 8 & 9 & 9 & 9 & 5 & 8 \\
\hline $\mathrm{SSV}(\mathrm{mg} / \mathrm{L})$ & 1 & 1 & 1 & 1 & 1 & 1 \\
\hline $\mathrm{N}-\mathrm{NH}_{3}(\mathrm{mg} / \mathrm{L})$ & 760 & 785 & 750 & 770 & 800 & 773 \\
\hline $\mathrm{N}-\mathrm{NH}_{3}$ soluble $(\mathrm{mg} / \mathrm{L})$ & 748 & 685 & 700 & 700 & 752 & 717 \\
\hline $\mathrm{N}$ total $(\mathrm{mg} / \mathrm{L})$ & 865 & 846 & 820 & 850 & 899 & 856 \\
\hline $\mathrm{N}$ total soluble $(\mathrm{mg} / \mathrm{L})$ & 787 & 774 & 755 & 782 & 802 & 780 \\
\hline $\mathrm{N}-\mathrm{NO}_{2}{ }^{-}(\mathrm{mg} / \mathrm{L})$ & 12.9 & 14 & 15.3 & 9.6 & 13.2 & 13 \\
\hline $\mathrm{N}-\mathrm{NO}_{2}{ }^{-}$soluble $(\mathrm{mg} / \mathrm{L})$ & 10.2 & 9.4 & 10.9 & 8.5 & 11.0 & 10 \\
\hline $\mathrm{N}-\mathrm{NO}_{3}{ }^{-}(\mathrm{mg} / \mathrm{L})$ & 14.8 & 14.6 & 18 & 10.9 & 16.7 & 15 \\
\hline $\mathrm{N}-\mathrm{NO}_{3}{ }^{-}$soluble $(\mathrm{mg} / \mathrm{L})$ & 11.1 & 12.6 & 15.2 & 8.6 & 12.5 & 12 \\
\hline NTK total $(\mathrm{mg} / \mathrm{L})$ & 840 & 820 & 790 & 830 & 869 & 830 \\
\hline NTK soluble $(\mathrm{mg} / \mathrm{L})$ & 768 & 754 & 730 & 760 & 780 & 758 \\
\hline
\end{tabular}

DQO: demanda química de oxígeno, $\mathrm{DBO}_{5}$ : demanda bioquímica de oxígeno a los 5 días, SST: sólidos suspendidos





CUADRO VI. LÍMITES MÁXIMOS PERMISIBLES SEGÚN LA NOM-001-SEMARNAT-1996

\begin{tabular}{lcc}
\hline Parámetros & \multicolumn{2}{c}{$\begin{array}{c}\text { Aguas costeras } \\
\text { Estuarios }\end{array}$} \\
\cline { 2 - 3 } & Promedio mensual & Promedio diario \\
\hline $\mathrm{SST}(\mathrm{mg} / \mathrm{L})$ & 75 & 125 \\
$\mathrm{DBO}_{5}(\mathrm{mg} / \mathrm{L})$ & 75 & 150 \\
$\mathrm{~N}_{\text {TOTAL }}(\mathrm{mg} / \mathrm{L})$ & 15 & 25 \\
\hline
\end{tabular}

SST: sólidos suspendidos totales, $\mathrm{DBO}_{5}$ : demanda bioquímica de oxígeno a los 5 días, $\mathrm{N}_{\text {TOT: }}$ nitrógeno total

$\mathrm{y}$ el $\mathrm{N}$ en forma soluble e insoluble. Las fracciones de la DQO son: $\mathrm{S}_{\mathrm{I}}=312 \mathrm{mg} / \mathrm{L}$ (DQO inerte soluble); $\mathrm{S}_{\mathrm{S}}=5688.5 \mathrm{mg} / \mathrm{L}(\mathrm{DQO}$ rápidamente biodegradable); $\mathrm{X}_{\mathrm{I}}=1192.8 \mathrm{mg} / \mathrm{L}$ (DQO inerte particulada), y $\mathrm{X}_{\mathrm{S}}=1981.8 \mathrm{mg} / \mathrm{L}$ (DQO lentamente biodegradable). Esta clasificación de la DQO (Han et al. 2015) indica que el substrato biodegradable alcanza el mayor porcentaje $\left(\mathrm{S}_{\mathrm{S}}+\mathrm{X}_{\mathrm{S}}\right)$ con un $83.6 \%$, por lo que la carga orgánica puede ser removida satisfactoriamente, lo cual ocurre actualmente en la planta de tratamiento. Nótese que la $\mathrm{S}_{\mathrm{S}}$ será removida rápidamente en un $62 \%$, luego será removida más lentamente la $\mathrm{X}_{\mathrm{S}}$ con un $21.6 \%$. La fracción lentamente biodegradable $\left(\mathrm{X}_{\mathrm{S}}\right)$, compuesta básicamente por partículas de alto peso molecular, coloides y sustrato orgánico particulado, debe pasar por una hidrólisis celular externa antes de estar disponible para su biodegradación (Gujer et al. 1995).

En el caso del substrato no biodegradable $\left(\mathrm{S}_{\mathrm{I}}+\right.$ $\mathrm{X}_{\mathrm{I}}$ ), éste alcanza solamente un $16.4 \%$ de la DQO total, $3.4 \%$ de $\mathrm{S}_{\text {I y }} 13 \%$ de $\mathrm{X}_{\mathrm{I}}$. Estas fracciones regularmente no son afectadas en un proceso de lodos activados. La fracción inerte soluble $\left(\mathrm{S}_{\mathrm{I}}\right)$ abandona la planta con el efluente del clarificador secundario mientras que la fracción inerte particulada $\left(\mathrm{X}_{\mathrm{I}}\right)$, se mezcla con el lodo purgado, contribuyendo a los sólidos suspendidos volátiles; de esta manera puede ser eliminada por adsorción (Fall et al. 2012). El fraccionamiento de la materia orgánica de las aguas residuales expresada como DQO fue inicialmente aplicado en aguas residuales municipales (Dulekgurgen et al. 2006, Fall et al. 2011); sin embargo, también se ha aplicado eficazmente en algunos tipos de aguas residuales industriales para definir las estrategias del tratamiento biológico a emplear, debido a que permite conocer las fracciones de la DQO que podrían ser degradadas por microorganismos (fracción biodegradable) y la fracción que permanece inerte (Karahan et al. 2008, Fall et al. 2012).

En el caso de las fracciones del nitrógeno total kjeldahl (NTK) se tienen los siguientes resultados: $\mathrm{S}_{\mathrm{TKN}}=1487.1 \mathrm{mg} / \mathrm{L}$ (NTK filtrado o soluble) y $\mathrm{X}_{\mathrm{TKN}}$ $=182.9 \mathrm{mg} / \mathrm{L}$ (NTK particulado o no soluble). Estos resultados indican un mayor porcentaje $(89 \%)$ de NTK soluble, el cual puede biodegradarse, en tanto que el NTK particulado (11\%) es difícil de eliminar, a 


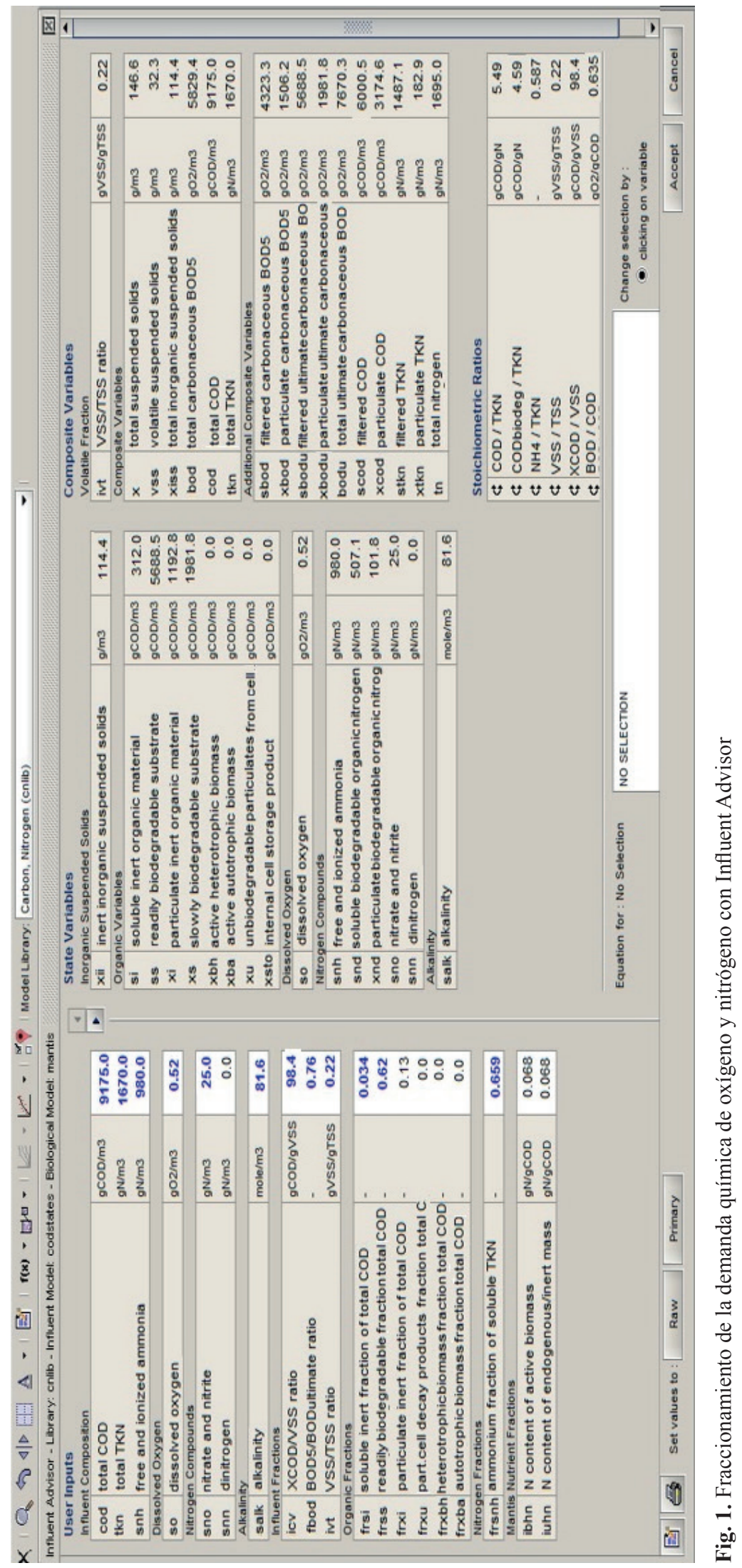


menos que exista una hidrólisis efectiva. Esto mismo se observa en la segunda columna del Influent Advisor con las fracciones biodegradables del nitrógeno: el nitrógeno orgánico biodegradable soluble $\left(\mathrm{S}_{\mathrm{ND}}\right)$, con $507.1 \mathrm{mg} / \mathrm{L}$, y el nitrógeno orgánico biodegradable particulado (X $\mathrm{X}_{\mathrm{ND}}$ ), con $101.8 \mathrm{mg} / \mathrm{L}$. Ambas fracciones se pueden eliminar adecuadamente, conforman un porcentaje del $62 \%$, respecto del nitrógeno amoniacal total $(980 \mathrm{mg} / \mathrm{L})$. El fraccionamiento del $\mathrm{N}$ (van Loosdrecht et al. 2016) se analiza a través del NTK, que es equivalente al nitrógeno orgánico más el nitrógeno amoniacal. Respecto a la remoción de nitrógeno en la PTAR en estudio, los resultados con Influent Advisor indican que el nitrógeno puede ser removido en un porcentaje mayor al $89 \%$ en forma de NTK, sólo que la capacidad de nitrificacióndesnitrificación de la planta existente es insuficiente, por lo cual será necesario hacer modificaciones o ampliaciones al sistema biológico.

\section{Escenarios de simulación \\ Calibración del modelo actual y variación de pará- metros que influyen en el proceso de nitrificación y desnitrificación}

El modelo utilizado para la calibración del sistema de tratamiento biológico de la planta en estudio se muestra en la figura 2. Los datos introducidos al simulador fueron la caracterización del influente (Cuadro IV), los diseños nominales de los equipos y la caracterización de lodos (Cuadro III), así como la relación XCOD/VSS (DQOPARTICULADA/SSV) obtenida en los reactores durante los cinco días de muestreo.

Los datos introducidos al programa GPS-X fueron: QINF $=190 \mathrm{~m}^{3} / \mathrm{d}$; reactor anóxico $\left(\mathrm{V}=133 \mathrm{~m}^{3}\right.$, $\mathrm{H}=4.1 \mathrm{~m}, \mathrm{~T}=38^{\circ} \mathrm{C}, \mathrm{OD}=0.29 \mathrm{mg} / \mathrm{L}$, alcalinidad $=$ $3073 \mathrm{mg} / \mathrm{L}, \mathrm{XCOD} / \mathrm{VSS}=0.4)$; CSTR $1\left(\mathrm{~V}=300 \mathrm{~m}^{3}\right.$, $\mathrm{H}=4.1 \mathrm{~m}, \mathrm{~T}=40^{\circ} \mathrm{C}, \mathrm{OD}=3.9 \mathrm{mg} / \mathrm{L}$, alcalinidad $=$ $2442 \mathrm{mg} / \mathrm{L}, \mathrm{XCOD} / \mathrm{VSS}=0.7) ; \mathrm{CSTR} 2\left(\mathrm{~V}=330 \mathrm{~m}^{3}\right.$, $\mathrm{H}=4.1 \mathrm{~m}, \mathrm{~T}=40^{\circ} \mathrm{C}, \mathrm{OD}=3.9 \mathrm{mg} / \mathrm{L}$, alcalinidad $=$ $2442 \mathrm{mg} / \mathrm{L}, \mathrm{XCOD} / \mathrm{VSS}=0.7, \mathrm{QRAS}_{\mathrm{R}}=50 \mathrm{~m}^{3} / \mathrm{d}$ ); MBR
$\left(\mathrm{V}=127 \mathrm{~m}^{3}\right.$, A SUP. MBR $_{\text {S }}=20.9 \mathrm{~m}^{2}$, A $_{\text {SUP. MEMBRANA }}$ $=1920 \mathrm{~m}^{2}, \mathrm{H}=4.1 \mathrm{~m}, \mathrm{~T}=39.1^{\circ} \mathrm{C}, \mathrm{OD}=3.9 \mathrm{mg} / \mathrm{L}$, alcalinidad $=2231 \mathrm{mg} / \mathrm{L}, \mathrm{XCOD} / \mathrm{VSS}=0.9$, flujo de aire cruzado $=15960 \mathrm{~m}^{3} / \mathrm{d}$, flujo de aire en tanque $=$ $15960 \mathrm{~m}^{3} / \mathrm{d}, \mathrm{QWAS}_{\mathrm{W}}=50 \mathrm{~m}^{3} / \mathrm{d}$ ).

Los resultados obtenidos se muestran en el cuadro VII. Se hace énfasis en los resultados de calidad del efluente permeado en el MBR. Con estos resultados, la concentración de SST en el reactor anóxico, CSTR1, CSTR2 y MBR fueron de $8297 \mathrm{mg} / \mathrm{L}$, $6775 \mathrm{mg} / \mathrm{L}, 5559 \mathrm{mg} / \mathrm{L}$ y $13290 \mathrm{mg} / \mathrm{L}$, respectivamente, mismos que comparados con los datos de operación normal de la planta (Cuadro III) representan una diferencia significativa, más en el caso del reactor anóxico (10 $454 \mathrm{mg} / \mathrm{L})$ y el MBR (7783 mg/L). En el caso de parámetros con magnitudes significativas (por ejemplo, los SST en el reactor aerobio), de manera general se puede decir que diferencias entre 5 y $15 \%$ de los valores simulados respecto a los datos medidos son considerados aceptables; por otra parte, en el caso de magnitudes pequeñas (por ejemplo, SST en el efluente), son aceptables diferencias de 10 a $100 \%$ comprendidas en concentraciones de alrededor de $1 \mathrm{mg} / \mathrm{L}$ (Shaw et al. 2012).

CUADRO VII. RESULTADOS DE LA SIMULACIÓN DE EFLUENTE PERMEADO COMPARADO CON DATOS ACTUALES DE LA PLANTA DE TRATAMENTO DE AGUAS RESIDUALES

\begin{tabular}{lcc}
\hline $\begin{array}{l}\text { Parámetros } \\
(\mathrm{mg} / \mathrm{L})\end{array}$ & $\begin{array}{c}\text { Efluente permeado } \\
\text { con simulación }\end{array}$ & $\begin{array}{c}\text { Efluente permeado. } \\
\text { Datos de laboratorio }\end{array}$ \\
\hline $\mathrm{SST}$ & 1.3 & 8.0 \\
$\mathrm{DBO}_{5}$ & 21.8 & 5.5 \\
$\mathrm{DQO}$ & 336.9 & 283.0 \\
$\mathrm{~N}_{\text {TOT }}$ & 1280.0 & 856.0 \\
$\mathrm{~N}^{-N H}$ & 546.4 & 773.0 \\
\hline
\end{tabular}

SST: sólidos suspendidos totales, $\mathrm{DBO}_{5}$ : demanda bioquímica de oxígeno a los 5 días, DQO: demanda química de oxígeno, $\mathrm{N}_{\text {Tот: }}$ nitrógeno total, $\mathrm{N}-\mathrm{NH}_{3}$ : nitrógeno amoniacal

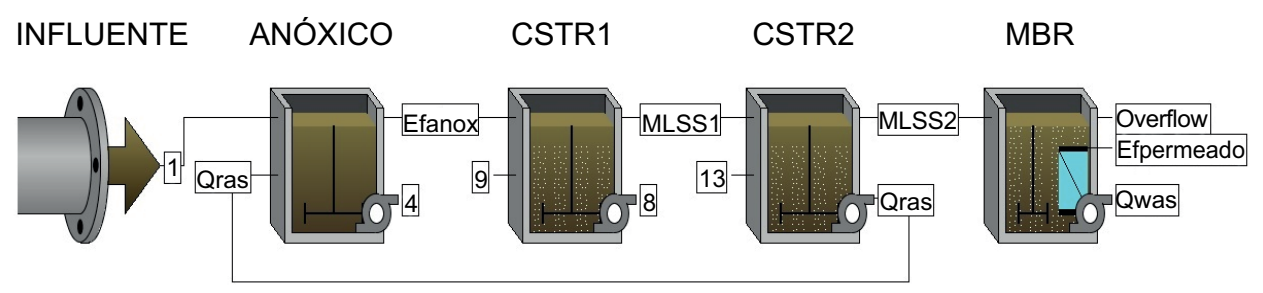

Fig. 2. Modelo del sistema de tratamiento biológico: reactor anóxico, reactores aerobios (CSTR) y reactor biológico de membrana (MBR) 
Debido a la diferencia de SST medidos y simulados en los reactores, se ajustó la relación XCOD/VSS con el fin de representar la operación actual del sistema biológico de tratamiento. Los resultados se muestran en el cuadro VIII. Con este ajuste en los reactores, los resultados del efluente permeado fueron los mismos que se muestran en el cuadro VII. Por otra parte, la relación F/M para los CSTR y MBR quedaron dentro del rango establecido en la bibliografía, al igual que el tiempo de retención celular (SRT), que fue de 18.8 d (de 5 a 20 d según Tchobanoglous et al. [2003]).

Una vez que se calibró el sistema de tratamiento actual, se hicieron cambios en algunos parámetros con el fin de disminuir la concentración del $\mathrm{N}_{\text {TOT }}$ en el efluente permeado. Se disminuyó el OD en los reactores a condiciones de operación normal (Tchobanoglous et al. 2003), ya que se consideran elevados y representan un gasto de energía impor- tante. El OD en el RANOX, CSTR1, CSTR2 y MBR se bajó a $0.0,2.0,2.0$ y $2.0 \mathrm{mg} / \mathrm{L}$, respectivamente, obteniendo con ello una concentración de $\mathrm{N}_{\text {TOT }}$ en el efluente de $1282 \mathrm{mg} / \mathrm{L}$ y una $\mathrm{DBO}_{5}$ de $21.66 \mathrm{mg} / \mathrm{L}$; es decir, disminuyendo el OD la nitrificación y desnitrificación se mantienen constantes. Cabe señalar que también se corrieron escenarios con cambios en la temperatura, alcalinidad, QRAS y QwAs, los cuales mostraron resultados similares con buena remoción de $\mathrm{DBO}_{5}$, pero no del $\mathrm{N}_{\text {Tот. }}$. Finalmente, se redujeron los SST en el RANOX, CSTR $1, \mathrm{CSTR}_{2}$ y se aumentaron en el MBR. La idea fue observar la calidad del efluente permeado ajustando los SST de acuerdo con los parámetros de operación manejados en la bibliografía. Se ajustaron los SST con la relación XCOD/ VSS (Cuadro IX).

Con este nuevo ajuste de XCOD/VSS se trataron de obtener los SST en el anóxico, CSTR1 y CSTR2 en el límite máximo de acuerdo con el rango de

CUADRO VIII. AJUSTE DE DEMANDA QUÍMICA DE OXÍGENO PARTICULADA / SÓLIDOS SUSPENDIDOS VOLÁTILES (XCOD/VSS) PARA CALIBRACIÓN DE SOLIDOS SUSPENDIDOS TOTALES (SST) DEL MODELO DE LA FIGURA 2 DE ACUERDO CON LA OPERACIÓN ACTUAL

\begin{tabular}{lcccc}
\hline & RANÓx & CSTR1 & CSTR2 & MBR \\
\hline XCOD/VSS. Datos de laboratorio & 0.40 & 0.70 & 0.70 & 0.90 \\
XCOD/VSS ajustado & 0.32 & 0.61 & 0.50 & 1.60 \\
OD. Datos de operación & 0.29 & 3.9 & 3.9 & 3.9 \\
SST (mg/L) con simulación & 10340 & 7757 & 7737 & 7668 \\
SST (mg/L). Datos de laboratorio & 10454 & 7783 & 7783 & 7783 \\
F/M (kg DBO5/kg MLVSS-d) & & 0.42 & 0.19 & 0.26 \\
F/M (Tchobanoglous et al. 2003) & & $0.2-0.6$ & $0.2-0.6$ & $0.1-0.4$ \\
\hline
\end{tabular}

XCOD/VSS: demanda química de oxígeno particulada / sólidos suspendidos volátiles, OD: oxígeno disuelto, SST: sólidos suspendidos totales, F/M: relación alimento/microorganismos o carga del lodo.

CUADRO IX. AJUSTE DE DEMANDA QUÍMICA DE OXÍGENO PARTICULADA / SÓLIDOS SUSPENDIDOS VOLÁTILES (XCOD/VSS) PARA DISMINUIR LA CONCENTRACIÓN DE SÓLIDOS SUSPENDIDOS TOTALES (SST) EN REACTORES DEL MODELO DE LA FIGURA 2 DE ACUERDO CON LA BIBLIOGRAFÍA

\begin{tabular}{lcccc}
\hline & RANÓx & CSTR1 & CSTR2 & MBR \\
\hline XCOD/VSS ajustado & 0.85 & 1.2 & 1.0 & 1.1 \\
OD (mg/L) & 0.0 & 2.0 & 2.0 & 2.0 \\
SST (mg/L) con simulación & 3965 & 3944 & 4026 & 10340 \\
SST (mg/L). Datos de laboratorio & 10454 & 7783 & 7783 & 7783 \\
SST (mg/L). (Tchobanoglous et al. 2003) & & $1500-4000$ & $1500-4000$ & $5000-20000$ \\
F/M (kg DBO5/kg MLVSS-d) & & 0.83 & 0.37 & 0.18 \\
\hline
\end{tabular}

XCOD/VSS: demanda química de oxígeno particulada / sólidos suspendidos volátiles, OD: oxígeno disuelto, SST: sólidos suspendidos totales, F/M: relación alimento/microorganismos o carga del lodo. 
operación normal (4000 mg/L), mientras que en el MBR arriba de los $10000 \mathrm{mg} / \mathrm{L}$ sin pasar de los 12 $000 \mathrm{mg} / \mathrm{L}$, que es el límite según el fabricante. Se manejaron un QRAS y un Qwas de $50 \mathrm{~m}^{3} / \mathrm{d}$. Con este ajuste de SST se obtuvieron concentraciones de SST, $\mathrm{DBO}_{5}$ y $\mathrm{N}_{\text {TOT }}$ en el efluente permeado de 1.096, 21.63 y $1283 \mathrm{mg} / \mathrm{L}$, respectivamente, cumpliendo con la NOM-001-SEMARNAT-1996 (SEMARNAT 1996) (Cuadro VI) en los dos primeros parámetros, pero no para el $\mathrm{N}_{\text {TOT. }}$ La relación F/M en el CSTR1 estuvo un poco por arriba del rango según la bibliografía, no así en el caso del CSTR2 y MBR. El SRT obtenido fue de $8.0 \mathrm{~d}$, también dentro de los límites marcados. El escenario simulado se muestra en la figura 3. Este escenario resultó el mejor en cuanto a operación de la PTAR actual, ya que, de inicio, al bajar la concentración de OD disminuye el consumo de energía; asimismo, al disminuir la concentración de SST en los reactores, se baja el costo de energía por aireación para mantener los sólidos en suspensión. En suma, ajustando los SST y el OD a los rangos de operación normal marcados en la bibliografía, se obtienen los mismos resultados que con concentraciones mayores. Sin embargo, considerando que el problema de nitrificación-desnitrificación persiste, se analizaron otros escenarios que pueden ayudar a resolver este problema.

\section{Modelo de ampliación con MBR}

Se propuso un nuevo reactor anóxico y la conversión del reactor anóxico existente a un CSTR, quedando entonces tres CSTR con los volúmenes actuales de la planta de tratamiento, más el MBR (Fig. 4).

Los datos introducidos al programa GPS-X (escenario default) fueron: $\mathrm{Q}_{\mathrm{INF}}=190 \mathrm{~m}^{3} / \mathrm{d}$; nuevo reactor anóxico $\left(\mathrm{V}=1000 \mathrm{~m}^{3}\right.$ [default], $\mathrm{T}=38^{\circ} \mathrm{C}$, $\mathrm{OD}=0.0 \mathrm{mg} / \mathrm{L}$, alcalinidad $=3073 \mathrm{mg} / \mathrm{L}, \mathrm{XCOD} /$ VSS $=1.48$ [default]); reactor anóxico (convertido a CSTR1) $\left(\mathrm{V}=133 \mathrm{~m}^{3}, \mathrm{H}=4.1 \mathrm{~m}, \mathrm{~T}=38^{\circ} \mathrm{C}, \mathrm{OD}=\right.$ $2.0 \mathrm{mg} / \mathrm{L}$, alcalinidad $=2442 \mathrm{mg} / \mathrm{L}, \mathrm{XCOD} / \mathrm{VSS}$ $=1.48$ [default]); reactor 1 (convertido a CSTR2) $\left(\mathrm{V}=300 \mathrm{~m}^{3}, \mathrm{H}=4.1 \mathrm{~m}, \mathrm{~T}=40^{\circ} \mathrm{C}, \mathrm{OD}=2.0 \mathrm{mg} / \mathrm{L}\right.$, alcalinidad $=2442 \mathrm{mg} / \mathrm{L}, \mathrm{XCOD} / \mathrm{VSS}=1.48[\mathrm{de}-$ fault]); reactor 2 (convertido a CSTR3) $\left(\mathrm{V}=330 \mathrm{~m}^{3}\right.$, $\mathrm{H}=4.1 \mathrm{~m}, \mathrm{~T}=40^{\circ} \mathrm{C}, \mathrm{OD}=2.0 \mathrm{mg} / \mathrm{L}$, alcalinidad $=2442 \mathrm{mg} / \mathrm{L}, \mathrm{QRAS}_{\mathrm{R}}=50 \mathrm{~m}^{3} / \mathrm{d}, \mathrm{XCOD} / \mathrm{VSS}=1.48$ [default]); MBR $\left(\mathrm{V}=127 \mathrm{~m}^{3}, \mathrm{~A}_{\text {SUP. MBR }}=20.9 \mathrm{~m}^{2}\right.$, ASUP. MEMBRANA $=1920 \mathrm{~m}^{2}, \mathrm{H}=4.1 \mathrm{~m}, \mathrm{~T}=39.1^{\circ} \mathrm{C}$, $\mathrm{OD}=2.0 \mathrm{mg} / \mathrm{L}$, alcalinidad $=2231 \mathrm{mg} / \mathrm{L}$, flujo de aire cruzado $=15960 \mathrm{~m}^{3} / \mathrm{d}$, flujo de aire en tanque $=15960 \mathrm{~m}^{3} / \mathrm{d}, \mathrm{Q}_{\mathrm{WAS}}=50 \mathrm{~m}^{3} / \mathrm{d}, \mathrm{XCOD} / \mathrm{VSS}=1.48$ [default]).
En el primer escenario simulado por default, los resultados obtenidos en el efluente permeado fueron: $\mathrm{SST}=0.90 \mathrm{mg} / \mathrm{L}, \mathrm{DBO}_{5}=32.07 \mathrm{mg} / \mathrm{L}, \mathrm{N}_{\mathrm{TOT}}$ $=1184 \mathrm{mg} / \mathrm{L}$. La concentración de $\mathrm{N}_{\mathrm{TOT}}$ disminuyó con relación al mejor escenario de operación actual de la PTAR (Fig. 3), pero la $\mathrm{DBO}_{5}$ aumentó. Las concentraciones de SST en los reactores fueron de $2311,3568,3151,2796$ y $9020 \mathrm{mg} / \mathrm{L}$ en el anóxico, CSTR1, CSTR2, CSTR3 y MBR, respectivamente, las cuales están muy alejadas de los datos de operación actual de la planta (Cuadro III).

En el siguiente escenario se ajustó la concentración de SST en los reactores con la relación XCOD/ VSS a las condiciones de operación actual de la PTAR. Los resultados se muestran en el cuadro $X$. Con este ajuste, los resultados de simulación en el efluente permeado fueron: $\mathrm{SST}=0.75 \mathrm{mg} / \mathrm{L}, \mathrm{DBO}_{5}$ $=32.76 \mathrm{mg} / \mathrm{L}, \mathrm{N}_{\mathrm{TOT}}=1177 \mathrm{mg} / \mathrm{L}$, muy similares al escenario por default. El SRT se elevó hasta $46.93 \mathrm{~d}$.

Para disminuir el SRT, se variaron el QRAS, QWAS y el volumen del reactor anóxico (pero con los mismos volúmenes de los CSTR, ya que estos últimos no pueden ampliarse porque su reconstrucción tendría un alto costo). Para mantener la concentración de SST en los reactores también se ajustó XCOD/ VSS de acuerdo con la bibliografía (Cuadro XI). El mejor escenario de operación se dio con el QRAS y el QwAs en $50 \mathrm{~m}^{3} / \mathrm{d}$, y con un volumen del reactor anóxico de $200 \mathrm{~m}^{3}$. Con este ajuste, los resultados de simulación en el efluente permeado fueron: $\mathrm{SST}=1.12 \mathrm{mg} / \mathrm{L}, \mathrm{DBO}_{5}=29.37 \mathrm{mg} / \mathrm{L}, \mathrm{N}_{\mathrm{TOT}}=1188 \mathrm{mg} / \mathrm{L}$, muy similares al escenario por default. El SRT disminuyó a 9.6 d. El escenario de simulación se presenta en la figura 5.

\section{Modelo de ampliación con clarificador secundario (CS)}

Se propuso un nuevo reactor anóxico y la conversión del reactor anóxico existente a un CSTR, quedando entonces tres CSTR con los volúmenes actuales de la planta de tratamiento, y se cambió el MBR por un clarificador secundario (CS) (Fig. 6).

Los datos introducidos en el programa GPS-X de inicio (escenario default) fueron: $Q_{\text {inf }}=190 \mathrm{~m}^{3} / \mathrm{d}$; nuevo reactor anóxico $\left(\mathrm{V}=1000 \mathrm{~m}^{3}\right.$ [default], $\mathrm{T}=38^{\circ} \mathrm{C}, \mathrm{OD}=0.0 \mathrm{mg} / \mathrm{L}$, alcalinidad $=3073 \mathrm{mg} / \mathrm{L}$, $\mathrm{XCOD} / \mathrm{VSS}=1.48$ [default]); reactor anóxico (convertido a CSTR 1$)\left(\mathrm{V}=133 \mathrm{~m}^{3}, \mathrm{H}=4.1 \mathrm{~m}, \mathrm{~T}\right.$ $=38^{\circ} \mathrm{C}, \mathrm{OD}=2.0 \mathrm{mg} / \mathrm{L}$, Alcalinidad $=2442 \mathrm{mg} / \mathrm{L}$, $\mathrm{XCOD} / \mathrm{VSS}=1.48$ [default]); reactor 1 (convertido a CSTR2) $\left(\mathrm{V}=300 \mathrm{~m}^{3}, \mathrm{H}=4.1 \mathrm{~m}, \mathrm{~T}=40^{\circ} \mathrm{C}, \mathrm{OD}=\right.$ $2.0 \mathrm{mg} / \mathrm{L}$, alcalinidad $=2442 \mathrm{mg} / \mathrm{L}, \mathrm{XCOD} / \mathrm{VSS}=$ 1.48 [default]); reactor 2 (convertido a CSTR3) $(\mathrm{V}=$ 


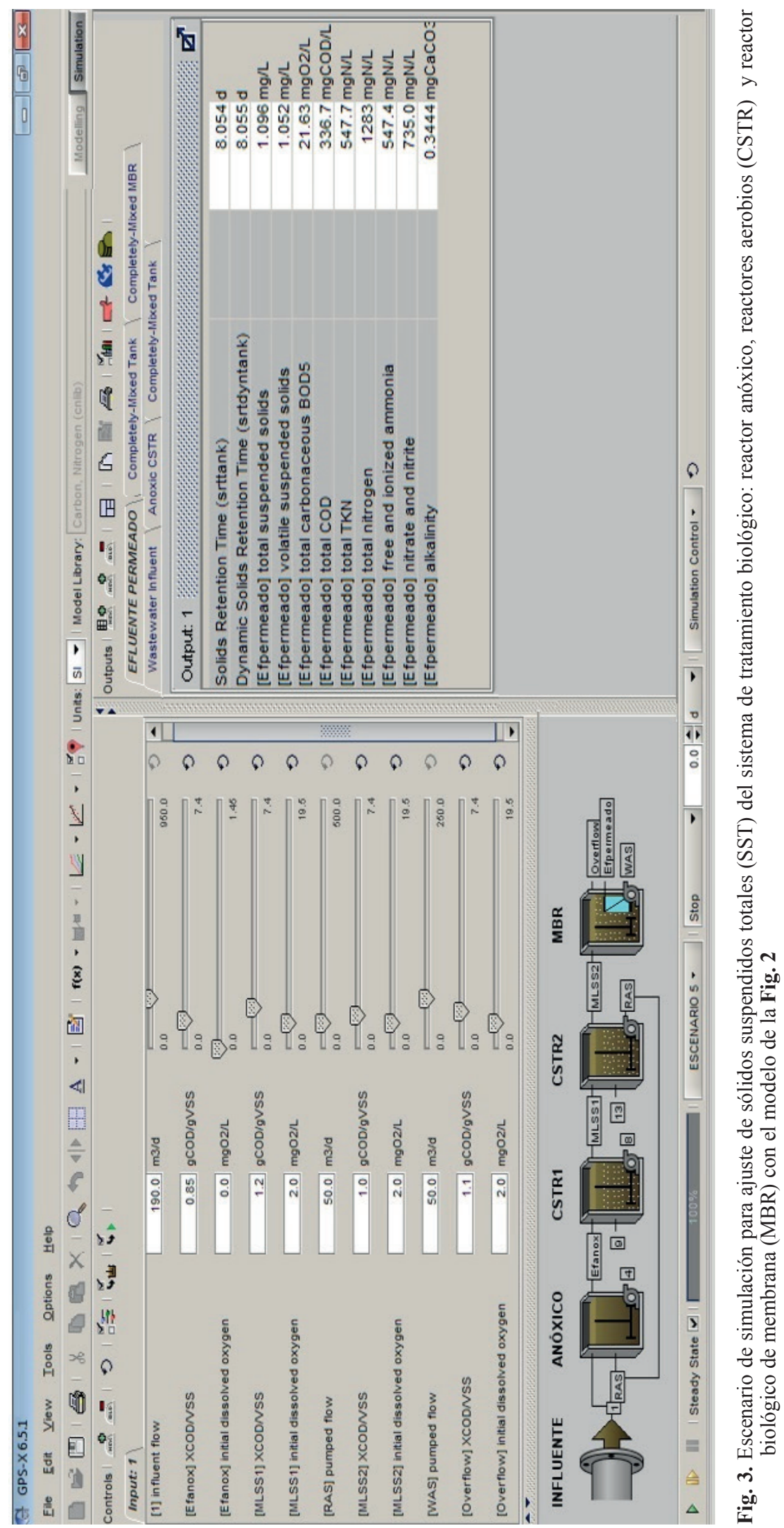




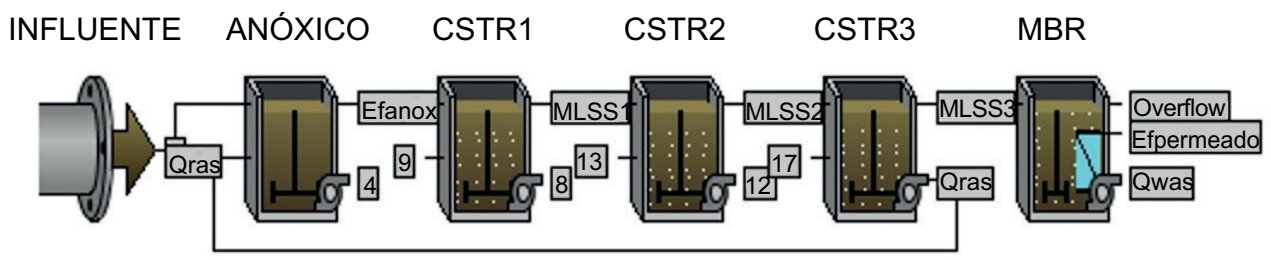

Fig. 4. Modelo de ampliación con reactor biológico de membrana (MBR)

CUADRO X. AJUSTE DE DEMANDA QUÍMICA DE OXÍGENO PARTICULADA / SÓLIDOS SUSPENDIDOS VOLÁTILES (XCOD/VSS) PARA LA CALIBRACIÓN DE LOS SÓLIDOS SUSPENDIDOS TOTALES (SST) DEL MODELO DE LA FIGURA 4 DE ACUERDO CON LA OPERACIÓN ACTUAL

\begin{tabular}{lccccc}
\hline & RANÓx & CSTR1 & CSTR2 & CSTR3 & MBR \\
\hline XCOD/VSS ajustado & 0.3 & 0.7 & 0.6 & 0.55 & 1.8 \\
OD (mg/L) & 0.0 & 2.0 & 2.0 & 2.0 & 2.0 \\
SST (mg/L) con simulación & 10950 & 7416 & 7604 & 7335 & 7494 \\
SST (mg/L). Datos de laboratorio & 10454 & 7783 & 7783 & 7783 & 7783 \\
F/M (kg DBO $/$ kg MLVSS-d) & & 0.98 & 0.25 & 0.18 & 0.29 \\
F/M (Tchobanoglous et al. 2003) & & $0.2-0.6$ & $0.2-0.6$ & $0.2-0.6$ & $0.1-0.4$ \\
\hline
\end{tabular}

XCOD/VSS: demanda química de oxígeno particulada / sólidos suspendidos volátiles, OD: oxígeno disuelto, SST: sólidos suspendidos totales, F/M: relación alimento/microorganismos o carga del lodo

CUADRO XI. AJUSTE DE DEMANDA QUÍMICA DE OXÍGENO PARTICULADA / SÓLIDOS SUSPENDIDOS VOLÁTILES (XCOD/VSS) PARA DISMINUIR LA CONCENTRACIÓN DE SÓLIDOS SUSPENDIDOS TOTALES (SST) EN REACTORES DEL MODELO DE LA FIGURA 4 DE ACUERDO CON LA BIBLIOGRAFÍA

\begin{tabular}{lccccc}
\hline & R ANÓx & CSTR1 & CSTR2 & CSTR3 & MBR \\
\hline XCOD/VSS ajustado & 0.85 & 1.3 & 1.15 & 1.0 & 1.2 \\
OD (mg/L) & 0.0 & 2.0 & 2.0 & 2.0 & 2.0 \\
SST (mg/L) con simulación & 4020 & 4148 & 4114 & 4167 & 11190 \\
SST (Tchobanoglous et al. 2003) & & $1500-4000$ & $1500-4000$ & $1500-4000$ & $5000-20000$ \\
F/M (kg DBO5/kg MLVSS-d) & & 1.78 & 0.49 & 0.34 & 0.20 \\
\hline
\end{tabular}

XCOD/VSS: demanda química de oxígeno particulada / sólidos suspendidos volátiles, OD: oxígeno disuelto, SST: sólidos suspendidos totales, F/M: relación alimento/microorganismos o carga del lodo

$330 \mathrm{~m}^{3}, \mathrm{H}=4.1 \mathrm{~m}, \mathrm{~T}=40^{\circ} \mathrm{C}, \mathrm{OD}=2.0 \mathrm{mg} / \mathrm{L}$, alcalinidad $=2442 \mathrm{mg} / \mathrm{L}, \mathrm{XCOD} / \mathrm{VSS}=1.48$ [default]); CS (tipo fondo plano, $\mathrm{A}_{\mathrm{SUP}}=100 \mathrm{~m}^{2}$ [default], $\mathrm{H}_{\text {agua }}$ $=3 \mathrm{~m}, Q_{W A S}=40 \mathrm{~m}^{3} / \mathrm{d}$ [default], QRAS $=2000 \mathrm{~m}^{3} / \mathrm{d}$ [default], alcalinidad $=2231 \mathrm{mg} / \mathrm{L}, \mathrm{XCOD} / \mathrm{VSS}=$ 1.48 [default]).

En el primer escenario simulado por default, los resultados obtenidos en el efluente fueron: $\mathrm{SST}=$ $8.73 \mathrm{mg} / \mathrm{L}, \mathrm{DBO}_{5}=2.22 \mathrm{mg} / \mathrm{L}$ y $\mathrm{N}_{\mathrm{TOT}}=126.5 \mathrm{mg} / \mathrm{L}$, reduciéndose significativamente la carga orgánica y el N Nот. La concentración de SST en los reactores fue de $8843,8819,8772$ y $8725 \mathrm{mg} / \mathrm{L}$ en el anóxico, CSTR1, CSTR2 y CSTR3 respectivamente, los cuales son muy similares a los datos de operación actual de la planta (Cuadro III) excepto en el reactor anóxico. Las relaciones $\mathrm{F} / \mathrm{M}$ estuvieron fuera del rango de operación según la bibliografía para todos los reactores (Cuadro X). El SRT fue de $17.78 \mathrm{~d}$ (entre tres y 15 d según Tchobanoglous et al. [2003]).

Observando los buenos resultados obtenidos en cuanto a remoción de $\mathrm{N}_{\text {TOT }}$ en el escenario por default, en el siguiente escenario se ajustaron los SST en los reactores con XCOD/VSS de acuerdo con lo que se maneja en la bibliografía. Se ajustó también el volumen del reactor anóxico, el cual se redujo hasta $800 \mathrm{~m}^{3}$. El área del CS se mantuvo en $100 \mathrm{~m}^{2}$, el QRAS se optimizó a $3000 \mathrm{~m}^{3} / \mathrm{d}$ y el QwAS a $100 \mathrm{~m}^{3} / \mathrm{d}$. Los resultados se muestran en cuadro XII. 









Fig. 6. Modelo de ampliación con clarificador secundario (CS)

CUADRO XII. AJUSTE DE DEMANDA QUÍMICA DE OXÍGENO PARTICULADA/ SÓLIDOS SUSPENDIDOS VOLÁTILES (XCOD/VSS) PARA DISMINUIR LA CONCENTRACIÓN DE SÓLIDOS SUSPENDIDOS TOTALES (SST) EN REACTORES DEL MODELO DE LA FIGURA 6 DE ACUERDO CON LA BIBLIOGRAFÍA

\begin{tabular}{lcccc}
\hline & R$_{\text {ANÓx }}$ & CSTR1 & CSTR2 & CSTR3 \\
\hline XCOD/VSS ajustado & 1.6 & 1.7 & 1.7 & 1.65 \\
OD (mg/L) & 0.0 & 2.0 & 2.0 & 2.0 \\
SST (mg/L) con simulación & 4083 & 3845 & 3824 & 3908 \\
SST (Tchobanoglous et al. 2003) & & $1500-4000$ & $1500-4000$ & $1500-4000$ \\
F/M (kg DBO $/$ kg MLVSS-d) & & 10.5 & 4.6 & 4.0 \\
F/M (Tchobanoglous et al. 2003) & & $0.2-0.6$ & $0.2-0.6$ & $0.2-0.6$ \\
\hline
\end{tabular}

XCOD/VSS: demanda química de oxígeno particulada / sólidos suspendidos volátiles, OD: oxígeno disuelto, SST: sólidos suspendidos totales, F/M: relación alimento/microorganismos o carga del lodo.

Con este ajuste, los resultados de simulación en el efluente fueron: $\mathrm{SST}=3.92 \mathrm{mg} / \mathrm{L}, \mathrm{DBO}_{5}=2.07 \mathrm{mg} / \mathrm{L}$ y $\mathrm{N}_{\text {TOT }}=89.49 \mathrm{mg} / \mathrm{L}$. El SRT fue de $7.32 \mathrm{~d}$ (entre tres y 15 d según Tchobanoglous et al. [2003]), mientras que la relación $\mathrm{F} / \mathrm{M}$ se mantuvo fuera del rango. Por otra parte, la carga hidráulica superficial (CHS) y la carga de sólidos (Cs) en el sedimentador secundario fueron de $0.9 \mathrm{~m}^{3} / \mathrm{m}^{2}$-d y $124.8 \mathrm{~kg} / \mathrm{m}^{2}$-d, respectivamente, las cuales son aceptables según la bibliografía (Tchobanoglous et al. 2003). Este escenario (Fig. 7) presentó los mejores resultados en cuanto a remoción de $\mathrm{N}_{\text {TOT }}$ con un $94.7 \%$, además de presentar una buena sedimentación de lodos, tal como se observa en la gráfica de barras.

Por último, considerando que la relación F/M está muy por arriba del rango de operación según la bibliografía, se simularon escenarios para disminuir dicha relación a través del $Q_{\text {RAS }}$, el QwAS y la relación XCOD/VSS. De esta manera, el QRAS y el Qwas se optimizaron en 1000 y $50 \mathrm{~m}^{3} / \mathrm{d}$, respectivamente, el volumen del reactor anóxico quedó en $800 \mathrm{~m}^{3}$ y el área del clarificador secundario se mantuvo en $100 \mathrm{~m}^{2}$. Los resultados se muestran en cuadro XIII. Con este ajuste, los resultados de simulación en el efluente fueron: $\mathrm{SST}=7.92 \mathrm{mg} / \mathrm{L}, \mathrm{DBO}_{5}=4.19 \mathrm{mg} / \mathrm{L}$ y $\mathrm{N}_{\mathrm{TOT}}$ $=258.5 \mathrm{mg} / \mathrm{L}$. El SRT fue de $13.28 \mathrm{~d}$ (entre tres y $15 \mathrm{~d}$ según Tchobanoglous et al. [2003]). Por otra parte, la carga hidráulica superficial (CHS) y la carga de sólidos (Cs) en el sedimentador secundario fueron de $1.4 \mathrm{~m}^{3} / \mathrm{m}^{2}-\mathrm{d}$ y $94.2 \mathrm{~kg} / \mathrm{m}^{2}-\mathrm{d}$, respectivamente, las cuales son aceptables según la bibliografía (Tchobanoglous et al. 2003). Con este escenario (Fig. 8) la remoción de $\mathrm{N}_{\text {TOT }}$ fue de un $84.8 \%$, además de presentar una buena sedimentación de lodos, tal como se observa en la gráfica de barras.

En suma, el proceso de desnitrificación llevado a cabo con el modelo de la figura $\mathbf{6}$ a través del reactor anóxico y el CS, llega a un punto en que el grado de reacción no puede aumentar, ya que está sujeto al proceso de nitrificación que se da en los tres CSTR. Si se quiere reducir aún más la concentración del $\mathrm{N}_{\text {TOT }}$, es necesario aumentar el volumen de los CSTR, lo cual generaría un costo extraordinario ajeno al objetivo de este trabajo de investigación. La idea principal fue reducir el $\mathrm{N}_{\text {TOT }}$ hasta un mínimo al menor costo posible, lo cual ocurrió con la propuesta de un nuevo reactor anóxico y el cambio del MBR por un CS. Cabe señalar que al aumentar el área superficial del CS se mantuvo el mismo grado de desnitrificación debido precisamente a la limitación del proceso de nitrificación en los reactores. Es posible llegar a este mismo escenario si se elimina el reactor anóxico y se 


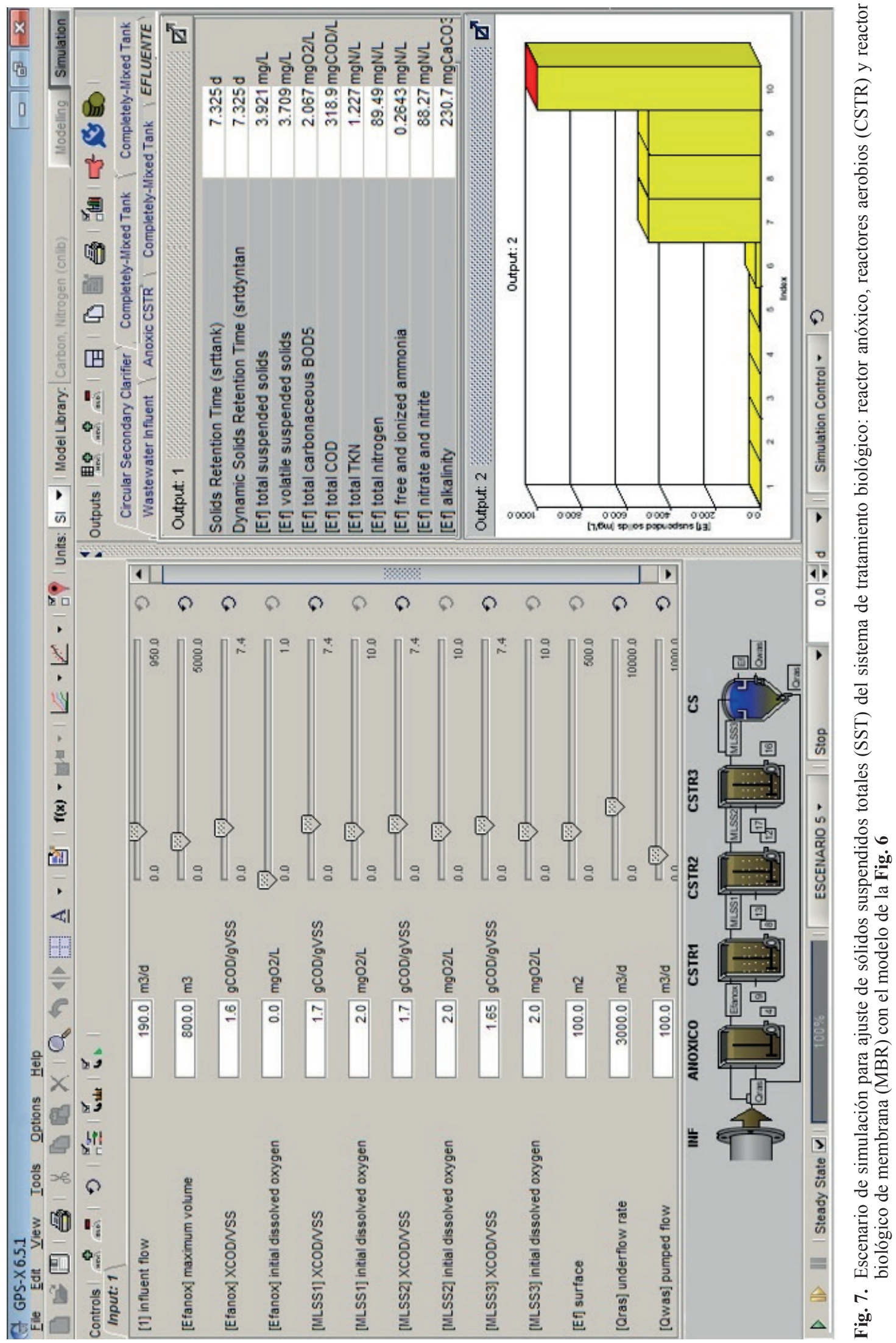


CUADRO XIII. AJUSTE DE LA RELACIÓN F/M (ALIMENTO/MICROORGANISMOS) PARA EL MODELO DE LA FIGURA 6

\begin{tabular}{lcccc}
\hline & R ANÓx & CSTR1 & CSTR2 & CSTR3 \\
\hline XCOD/VSS ajustado & 1.2 & 1.4 & 1.4 & 1.4 \\
OD (mg/L) & 0.0 & 2.0 & 2.0 & 2.0 \\
SST (mg/L) con simulación & 10460 & 7827 & 7733 & 7917 \\
SST (Tchobanoglous et al. 2003) & & $1500-4000$ & $1500-4000$ & $1500-4000$ \\
F/M (kg DBO $/$ kg MLVSS-d) & & 2.4 & 1.0 & 0.8 \\
F/M (Tchobanoglous et al. 2003) & & $0.2-0.6$ & $0.2-0.6$ & $0.2-0.6$ \\
\hline
\end{tabular}

XCOD/VSS: demanda química de oxígeno particulada / sólidos suspendidos volátiles, OD: oxígeno disuelto, SST: sólidos suspendidos totales, F/M: relación alimento/microorganismos o carga del lodo.

aumenta el área superficial del CS, ya que este último se opera en condiciones anóxicas; sin embargo, esto generaría mayor costo de construcción y uso de terreno al utilizarse más clarificadores secundarios.

\section{CONCLUSIONES}

El sistema de ampliación con clarificador secundario resultó la mejor alternativa para la remoción de nitrógeno, carga orgánica y sólidos. En el caso de la eliminación de $\mathrm{N}_{\mathrm{TOT}}$, el mejor escenario de remoción alcanza un $94.7 \%$; sin embargo, la relación F/M se mantiene muy lejos del rango de operación marcado en la bibliografía. Al hacer el ajuste de la $\mathrm{F} / \mathrm{M}$ a un rango aceptable, la remoción de $\mathrm{N}_{\text {TOT }}$ se reduce a un $84.8 \%$. En ambos casos la sedimentación de lodos es muy buena. En este sentido, la relación F/M, aunque influye en el proceso de nitrificación, puede ser manejada fuera del rango de operación, siempre y cuando la sedimentación en el clarificador secundario sea aceptable.

Aun cuando la concentración de $\mathrm{N}_{\mathrm{TOT}}$ en los escenarios de ampliación con clarificador secundario no cumple con los límites máximos permisibles (LMP) de descarga según la NOM-001-SEMARNAT-1996 (SEMARNAT 1996), este nitrógeno puede disminuirse en la etapa de tratamiento terciario con el que cuenta la planta de tratamiento en estudio, hasta llevarlo dentro de los LMP.

Haciendo una comparación de remoción de carga orgánica, nitrógeno y sólidos del mejor escenario de ampliación con MBR y CS, se puede observar que en el caso del MBR se alcanzó una remoción de $99.4 \%$ en $\mathrm{DBO}_{5}, 99.2 \%$ en SST y $30 \%$ en $\mathrm{N}_{\text {TOT }}$, mientras que en el caso de la ampliación con CS se alcanzó una remoción de $99.9 \%$ en $\mathrm{DBO}_{5}, 97.3 \%$ en SST y $94.7 \%$ en $\mathrm{N}_{\text {TOT. }}$.

\section{NOMENCLATURA}

CS: clarificador secundario

CSTR: reactor aerobio tipo mezcla completa

$\mathrm{DBO}_{5}$ : demanda bioquímica de oxígeno a los 5 días

DQO: demanda química de oxígeno

Ef: efluente

Efanox: efluente de reactor anóxico

Efpermeado: efluente permeado del MBR

F/M: relación alimento/microorganismos o carga del lodo. Relación que existe entre la cantidad de materia orgánica o alimento que ingresa al sistema biológico (F) y la cantidad de microorganismos presentes en el sistema biológico $(\mathrm{M})$

fbod: relación de $\mathrm{DBO}_{5}$ y $\mathrm{DBO}$ última

frsi: fracción de DQO inerte soluble

frss: fracción de $\mathrm{DQO}$ rápidamente biodegradable

frxi: fracción de DQO inerte particulada

frsnh: fracción de nitrógeno amoniacal y nitrógeno total Kjeldahl solubles

icv: relación de DQO particulada y sólidos suspendidos volátiles

ivt: relación de sólidos suspendidos volátiles (SSV) y sólidos suspendidos totales (SST)

MBR: reactor biológico de membrana

MLSS: sólidos suspendidos totales en el licor mezclado

MLVSS: sólidos suspendidos volátiles en el licor mezclado

$\mathrm{N}-\mathrm{NH}_{3}$ : nitrógeno amoniacal

$\mathrm{N}-\mathrm{NO}_{2}^{-}$: nitrógeno como nitritos

$\mathrm{N}-\mathrm{NO}_{3}{ }^{-}$: nitrógeno como nitratos

NTK: nitrógeno total Kjeldahl

$\mathrm{N}_{\text {TOт: nitrógeno total }}$

Overflow: sobrenadante del MBR

P: fósforo

PTAR: planta de tratamiento de aguas residuales

QINF: flujo de influente 


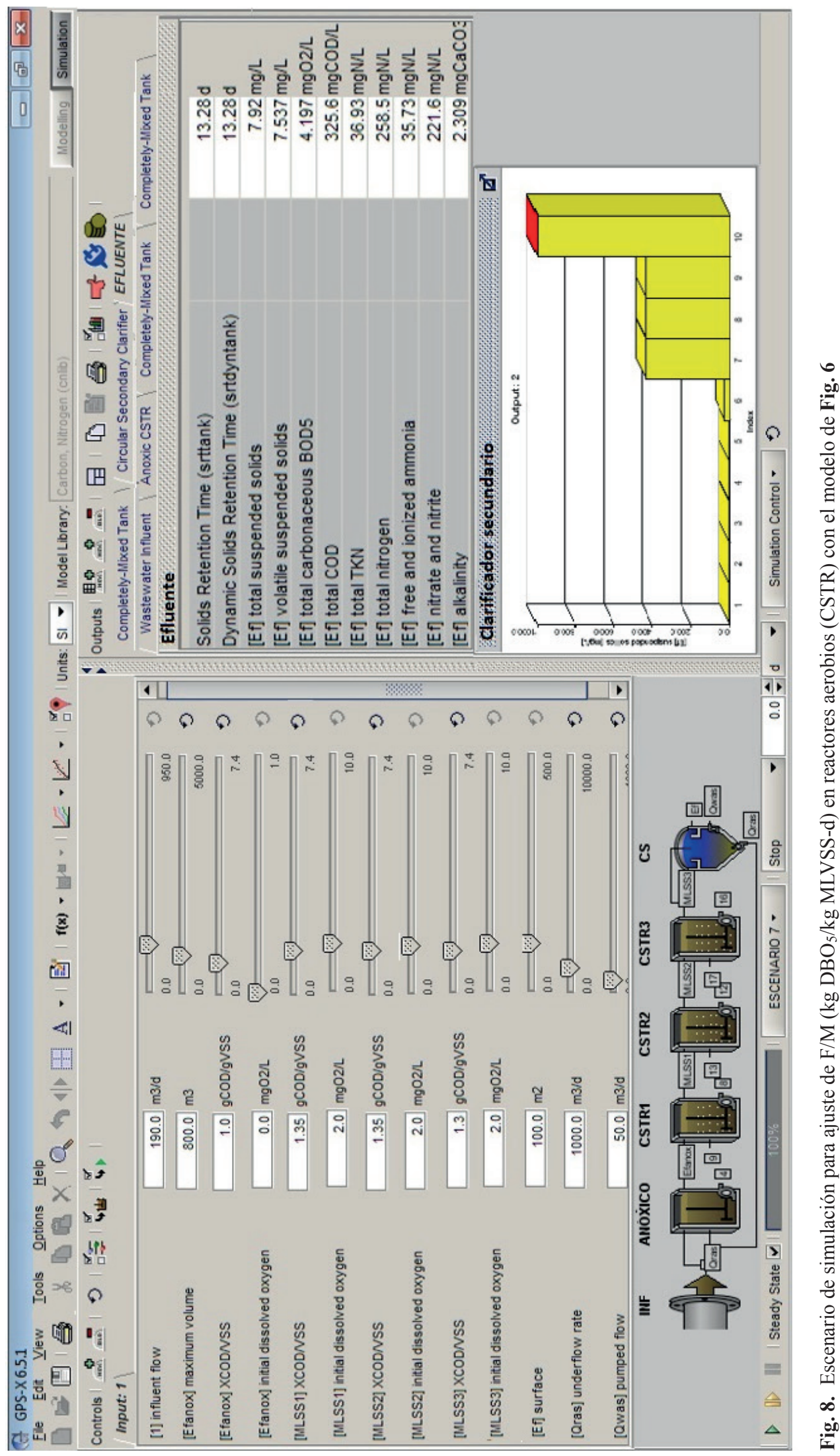


QRAS: flujo de recirculación

QwAs: flujo de purga

$\mathrm{S}_{\mathrm{I}}$ : DQO inerte soluble

$\mathrm{S}_{\mathrm{NH}}$ : nitrógeno amoniacal soluble

$\mathrm{S}_{\mathrm{ND}}$ : nitrógeno orgánico soluble biodegradable

SRT: tiempo de retención de sólidos

$\mathrm{S}_{\mathrm{S}}$ : DQO fácilmente biodegradable

SST: sólidos suspendidos totales

SSV: sólidos suspendidos volátiles

XCOD/VSS: DQOPARTICULADA/Sólidos suspendidos

volátiles

$\mathrm{X}_{\mathrm{I}}$ : DQO inerte en suspensión

$\mathrm{X}_{\mathrm{S}}$ : DQO lentamente biodegradable

$\mathrm{X}_{\mathrm{ND}}$ : nitrógeno orgánico particulado biodegradable

\section{REFERENCIAS}

APHA (2005). Standard methods for the examination de water and wastewater. Seventeenth ed. American Public Health Association, American Water Works Association, Water Pollution Control Federation, Washington D.C., 1364 pp.

Bhadra S., Narvaez C., Thomson D.J. y Bridges G.E. (2015). Non-destructive detection of fish spoilage using a wireless basic volatile sensor. Talanta 134, 718-723. DOI: 10.1016/j.talanta.2014.12.017

Dulekgurgen E., Dogruel S., Karahan Ö. y Orhon D. (2006). Size distribution of wastewater COD fractions as an index for biodegradability. Water Res. 40, 273282. DOI: $10.1016 /$ j.watres.2005.10.032

Espinosa M.A., Bravo O., Ortega J.M. e Hidalgo A. (2014). Evaluación de la nitrificación a través de perfiles operacionales en un reactor aerobio. Ingenierías XVII (62), 50-59.

Espinosa M.A., Ruiz T., Hidalgo A. y Delgado R. (2019). Efecto de la carga hidráulica de un filtro percolador en el proceso de nitrificación. Rev. Mex. Ing. Quím. 18 (1), 107-113. DOI: 10.24275/uam/izt/dcbi/ revmexingquim/2019v18n1/Espinosa

Fall C. Flores A.N., Espinosa M.A., Vázquez G., Loaiza N.J., van Loosdrecht M.C.M. y Hooijmans C.M. (2011). Divergence between respirometry and physicochemical methods in the fractionation of the chemical oxygen demand in municipal wastewater. Water Environ. Res. 83 (2), 162-172. DOI: $10.2175 / 106143010 X 12780288627931$

Fall C., Millán E., Bâ K.M., Gallego I., García D., Díaz C. y Solís C. (2012). COD fractionation and biological treatability of mixed industrial wastewaters. J. Environ. Manage. 113, 71-77. DOI: 10.1016/j.jenvman.2012.08.032
Gujer W., Henze M., Mino T., Matsuo T., Wentzel M. y Marais G. (1995). The activated sludge model No. 2: Biological phosphorus removal. Water Sci. Technol. $31(2), 1-11$. DOI: 10.1016/0273-1223(95)00175-M

Han N., Hao H., Urase T. y Yew-Hoong K. (2015). A critical review on characterization strategies of organic matter for wastewater and water treatment process. Bioresource Technol. 193, 523-533.

DOI: 10.1016/j.biortech.2015.06.091

Hydromantis (2014). Software GPS-X. Environmental Software Solutions, INC. Applications [en línea]. https;//www.hydromantis.com/GPSX-applications. html 01/08/2017.

Karahan Ö., Dogruel S., Dulekgurgen E. y Orhon D. (2008). COD fractionation of tannery wastewaters. Particle size distribution, biodegradability and modeling. Water Res. 42, 1083-1092.

DOI: 10.1016/j.watres.2007.10.001

Tchobanoglous G., Burton F. y Stensel H. (2003). Wastewater engineering, treatment and reuse. Fourth edition. Mc.Graw Hill, Inc., Nueva York, EUA, 1819 pp.

SEMARNAT (1996). Norma Oficial Mexicana NOM001-SEMARNAT-1996. Límites máximos permisibles de contaminantes en las descargas de aguas residuales en aguas y bienes nacionales. Secretaría de Medio Ambiente y Recursos Naturales. Diario Oficial de la Federación, 23 de abril de 2003.

Pratyush B., Saroj K. y Arun K. (2014). Evaluation of poultry viscera as potential fish feed ingredient, compared to fishmeal. Int. J. Current Res. 6 (2), 5241-5243.

Radjenović J., Matošić M., Mijatović I., Petrović M. y Barceló D. (2008). Membrane bioreactor (MBR) as an advanced wastewater treatment technology. Environ. Chem. 5, 37-101.

DOI: $10.1007 / 698 \_5093$

Shaw A., Langergraber G., Reiger L., Winkler S., Gillot S. y Ohtsuki T. (2012). Guidelines for using activated sludge models. First edition. IWA Publishing. Londres, Inglaterra, $269 \mathrm{pp}$.

Van Loosdrecht M., López C., Meijer S., Hooijmans C. y Brdjanovic D. (2015). Twenty-five years of ASM1: past, present and future of wastewater treatment modelling. J. Hydroinform. 17 (5), 697-718.

DOI: 10.2166 /hydro.2015.006

Van Loosdrecht M., Nielsen P., López C. y Brdjanovic D. (2016). Experimental methods in wastewater treatment. First edition. IWA publishing. Londres, Inglaterra, $319 \mathrm{pp}$. 